\title{
2+1 flavor lattice QCD with Lüscher's Domain-Decomposed HMC algorithm
}

\author{
PACS-CS Collaboration: Y. Kuramashi ${ }^{* a, b \dagger}$, S. Aoki ${ }^{a, c}$, K.-I. Ishikawa $^{d}$, T. Ishikawa ${ }^{b}$, \\ N. Ishizuka ${ }^{a, b}$, K. Kanaya $^{a}$, N. Tsutsui ${ }^{e}$, M. Okawa ${ }^{d}$, Y. Taniguchi ${ }^{a, b}$, A. Ukawa ${ }^{a, b}$, \\ T. Yoshié , $^{a, b}$ \\ ${ }^{a}$ Graduate School of Pure and Applied Sciences, University of Tsukuba, Tsukuba, Ibaraki \\ 305-8571, Japan \\ ${ }^{b}$ Center for Computational Sciences, University of Tsukuba, Tsukuba, Ibaraki 305-8577, Japan \\ ${ }^{c}$ Riken BNL Research Center, Brookhaven National Laboratory, Upton, New York 11973, USA \\ ${ }^{d}$ Department of Physics, Hiroshima University, Higashi-Hiroshima, Hiroshima 739-8526, Japan \\ ${ }^{e}$ High Energy Accelerator Research Organization (KEK), Tsukuba 305-0801, Japan
}

\begin{abstract}
We report on a study of $2+1$ flavor lattice QCD with the $O(a)$-improved Wilson quarks on a $16^{3} \times 32$ lattice at the lattice spacing $1 / a \approx 2 \mathrm{GeV}$ employing Lüscher's domain-decomposed HMC(LDDHMC) algorithm. This is dedicated to a preliminary study for the PACS-CS project which plans to complete the Wilson-clover $N_{f}=2+1$ program lowering the up-down quark masses close to the physical values as much as possible. We focus on three issues: (i) how light quark masses we can reach with LDDHMC, (ii) efficiency of the algorithm compared with the conventional HMC, (iii) parameter choice for the production runs on PACS-CS.
\end{abstract}

XXIVth International Symposium on Lattice Field Theory

July 23-28, 2006

Tucson, Arizona, USA

\footnotetext{
* Speaker.

${ }^{\dagger}$ E-mail: kuramasi@het.ph.tsukuba.ac.jp
} 


\section{Introduction}

In the past decade the progress of simulation algorithms and the availability of more affluent computational resources have enabled us to investigate two- and three-flavor dynamical quark effects with lighter quark masses. The CP-PACS and JLQCD joint project[1] has performed a $2+1$ flavor full QCD simulation employing the $O(a)$-improved Wilson quark action with nonperturbative $c_{\mathrm{SW}}$ and the Iwasaki gauge action[2] on a $(2 \mathrm{fm})^{3}$ box at three lattice spacings. Unfortunately, the lightest up and down quark mass reached is about $64 \mathrm{MeV}$ corresponding to $m_{\pi} / m_{\rho} \approx 0.6$.

A goal of the next decade should be the realistic full QCD simulation with the physical up, down and strange quark masses. The PACS-CS project, which is based on the PACS-CS (Parallel Array Computer System for Computational Sciences) computer installed at University of Tsukuba on July 1 of 2006[3], aims at this goal succeeding the Wilson-clover $2+1$ program of the previous CP-PACS/JLQCD project.

In this report we present a preparatory study for the PACS-CS project using $(1.6 \mathrm{fm})^{3}$ lattice. To simulate the dynamical up and down quarks, we employ the domain-decomposed HMC algorithm proposed by Lüscher[4]. The effectiveness of this algorithm for small quark mass region is already shown in the two-flavor case [4, 5]. The strange quark is included by the exact Polynomial HMC (PHMC) algorithm[6]. Our primary purpose is to investigate how light up and down quark masses we can go down to with LDDHMC. The efficiency of LDDHMC is compared to that of the conventional HMC. Based on this study we finally make a parameter choice for the production runs on PACS-CS, and briefly discuss the physics plan.

\section{Simulation details}

We employ the $O(a)$-improved Wilson quark action with nonperturbative $c_{\mathrm{SW}}[7]$ and the Iwasaki gauge action at $\beta=1.9$ on a $16^{3} \times 32$ lattice. The lattice spacing is about $0.1 \mathrm{fm}$ from the previous CP-PACS/JLQCD results on a $20^{3} \times 40$ lattice[1]. In Table 1 we summarize the details of the simulation parameters. We employ four degenerate up and down quark masses based on the previous CP-PACS/JLQCD results, while the strange quark mass is fixed at $\kappa_{\mathrm{s}}=0.1364$ close to the physical point $\kappa_{\mathrm{s}}=0.136412(50)[1]$. The LDDHMC algorithm is implemented with a $8^{4}$ block size, while the exact PHMC algorithm is not domain-decomposed. We choose the trajectory length of $\tau=0.5 / \sqrt{2}$. With the integers $N_{0}, N_{1}, N_{2}$ the step sizes are given by $\delta \tau=\tau /\left(N_{0} N_{1} N_{2}\right)$ for the gauge part, $\delta \tau=\tau /\left(N_{1} N_{2}\right)$ for the strange quark and the UV part of the up and down quarks, and $\delta \tau=\tau / N_{2}$ for the IR part of the up and down quarks. The Wilson quark matrix inversion is carried out by the even/odd preconditioned BiCGStab solver with the stopping condition $|D x-b| /|b|<10^{-9}$ for the force calculation and $10^{-14}$ for the Hamiltonian.

All the simulations presented in this report are carried out on Hitachi SR11000/J1 at Information Technology Center of the University of Tokyo.

\section{Numerical results}

\subsection{History of $d H$}

In Fig 1 we show the history of $d H$ for each $\kappa_{\text {ud }}$. We observe a frequent occurrence of spikes 


\begin{tabular}{|c|c|c|c|c|}
\hline$\kappa_{\mathrm{ud}}$ & 0.13700 & 0.13741 & 0.13759 & 0.13770 \\
\hline$N_{0}, N_{1}, N_{2}$ & $4,5,6$ & $4,5,8$ & $4,5,12$ & $4,5,14$ \\
\hline$N_{\text {poly }}$ & 130 & 140 & 140 & 140 \\
\hline thermalization & 2000 & 1700 & 1600 & 1500 \\
\hline No. traj. & 2000 & 2000 & 2000 & 900 \\
\hline bin size[trajs] & 50 & 50 & 50 & 50 \\
\hline$\langle P\rangle$ & $0.569126(35)$ & $0.569921(37)$ & $0.570341(43)$ & $0.570574(79)$ \\
\hline$P_{\text {spike }}(|d H|>2)$ & 0 & 0.002 & 0.018 & 0.017 \\
\hline$P_{\text {spike }}(|d H|>10)$ & 0 & 0.0005 & 0.0105 & 0.008 \\
\hline$P_{\text {spike }}(|d H|>100)$ & 0 & 0 & 0.002 & 0.003 \\
\hline$\left\langle\mathrm{e}^{-d H}\right\rangle$ & $1.003(6)$ & 0.9995(99) & $0.9867(92)$ & $0.975(13)$ \\
\hline$P_{\mathrm{acc}}(\mathrm{HMC})$ & $0.88(1)$ & $0.86(1)$ & $0.89(1)$ & $0.86(2)$ \\
\hline$P_{\text {acc }}(\mathrm{GMP})$ & $0.94(1)$ & $0.95(1)$ & $0.94(1)$ & $0.93(1)$ \\
\hline \#mult/traj & $20284(61)$ & $31776(171)$ & 53403(395) & 69342(990) \\
\hline$\tau_{\text {int }}[P]$ & $10.6(2.7)$ & $16.0(6.6)$ & $18.0(6.0)$ & $36.8(23.6)$ \\
\hline$\tau_{\text {int }}[P] \cdot \#$ mult $/ 10^{3}$ & 215(55) & $508(210)$ & $961(320)$ & 2552(1636) \\
\hline No. config. & 200 & 200 & 200 & 90 \\
\hline bin size[configs] & 5 & 5 & 5 & 5 \\
\hline fitting range $\left[t_{\min }, t_{\max }\right]$ & {$[8,13]$} & {$[8,13]$} & {$[7,12]$} & {$[7,12]$} \\
\hline$m_{P S}\left(\kappa_{\mathrm{ud}}\right)$ & $0.3303(21)$ & $0.2509(29)$ & $0.185(5)$ & $0.158(8)$ \\
\hline$m_{P S}\left(\kappa_{\mathrm{ud}}\right)[\mathrm{MeV}]$ & $655(4)$ & $498(6)$ & $367(9)$ & 313(16) \\
\hline$m_{u d}^{\mathrm{AWI}}[\mathrm{MeV}]$ & $63.7(4)$ & $34.8(5)$ & $20.7(5)$ & $15.2(12)$ \\
\hline$m_{\mathrm{PS}}^{2} / m_{\mathrm{ud}}^{\mathrm{AWI}}$ & $3.39(3)$ & $3.59(7)$ & $3.27(14)$ & $3.27(23)$ \\
\hline No. config. & 200 & 200 & 200 & 90 \\
\hline bin size[configs] & 5 & 5 & 5 & 5 \\
\hline$\mu_{\text {median }}$ & 0.02018 & 0.01077 & 0.00718 & 0.00602 \\
\hline$\mu_{\text {average }}$ & $0.01982(24)$ & $0.01059(22)$ & $0.00716(14)$ & $0.00619(26)$ \\
\hline$\sigma$ & $0.00209(16)$ & $0.00203(15)$ & $0.00166(15)$ & $0.00138(21)$ \\
\hline$\sigma \sqrt{V} / a$ & $0.755(60)$ & $0.735(53)$ & $0.601(54)$ & $0.501(76)$ \\
\hline
\end{tabular}

Table 1: Simulation parameters and basic physical quantities. \#mult is the number of multiplications of the Wilson-Dirac quark matrix on the full lattice including even and odd sites.

for $\kappa_{\mathrm{ud}}=0.13759$ and 0.13770 , whose probabilities for $|d H|>2$ are about $2 \%$. Although the acceptance ratios for $\kappa_{\mathrm{ud}}=0.13759$ and 0.13770 are still sufficiently high, $\left\langle\mathrm{e}^{-d H}\right\rangle$ which measures the area preserving property deviates from unity by $2 \sigma$. It is clear that the spikes are a potential source to violate the area preserving property. In order to avoid any problem associated with the spikes, we shall incorporate the replay trick $[4,8]$ in the production run.

\subsection{Magnitude of force terms}

Figure 2 shows the magnitude of the four force terms as a function of the axial vector Ward identity quark mass $m_{\mathrm{ud}}^{\mathrm{AWI}}$ discussed below; $F_{g}$ denotes the gauge part, $F_{\mathrm{UV}, \mathrm{IR}}$ for the UV and IR parts of the up and down quarks, and $F_{S}$ for the strange quark. Note that the definition of the 

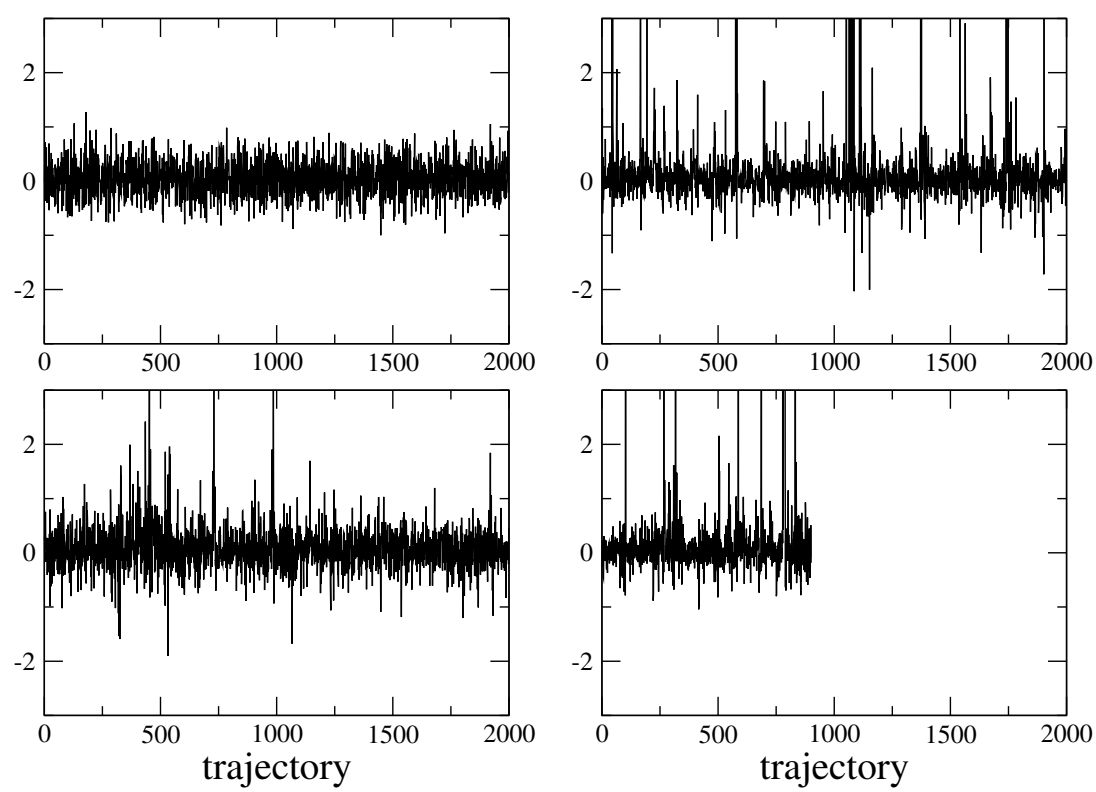

Figure 1: Time histories of $d H$ as a function of the trajectory number after thermalization for $\kappa_{\text {ud }}=$ 0.13700(top left), 0.13741(bottom left), 0.13759(top right) and 0.13770(bottom right).

magnitude of force term is a factor two larger than that in Ref.[4]. We observe that $\left\|F_{g}\right\|,\left\|F_{\mathrm{UV}}\right\|$ and $\left\|F_{s}\right\|$ are almost independent of the quark mass, while $\left\|F_{\mathrm{IR}}\right\|$ gradually increases as the up and down quark masses decreases. At $\kappa_{\mathrm{ud}}=0.13770$ we find

$$
\left\|F_{g}\right\|:\left\|F_{\mathrm{UV}}\right\|:\left\|F_{\mathrm{s}}\right\|:\left\|F_{\mathrm{IR}}\right\| \approx 24: 6: 3: 1
$$

This result suggests that our choice of $\delta \tau$ for the strange quark may be unnecessarily fine.

\subsection{Comparison of LDDHMC and HMC}

For a direct comparison between LDDHMC and HMC we have repeated the $2+1$ flavor simulation at $\kappa_{\mathrm{ud}}=0.13700$ and $\kappa_{\mathrm{s}}=0.13640$ with the conventional HMC algorithm employing the same parameters except $\tau=1$ for HMC. Analyzing 3000 trajectories after thermalization we obtain $\tau_{\text {int }}[P]=6.6(1.8)$ and \#mult/traj=148625(626) for HMC. Since the trajectory lengths are differently chosen for LDDHMC and HMC, we compare their efficiency by $\tau_{\text {int }}[P] \cdot \#$ \#ult $/ 10^{3}$ which should be independent of the trajectory length. This quantity is found to be 215(55) for LDDHMC and 981(268) for HMC. Hence LDDHMC is almost five times more efficient than HMC at $m_{\mathrm{PS}} / m_{\mathrm{V}} \approx 0.6$. We should keep in mind that this comparison involves the strange quark part simulated by the exact PHMC algorithm.

\subsection{Quark masses}

The up and down quark masses are measured by using the axial vector Ward identity (AWI):

$$
m_{\mathrm{ud}}^{\mathrm{AWI}}=\frac{Z_{A}}{Z_{P}} \frac{\left\langle 0\left|\nabla_{4} A_{4}^{\mathrm{impr}}\right| P S\right\rangle}{2\langle 0|P| P S\rangle},
$$




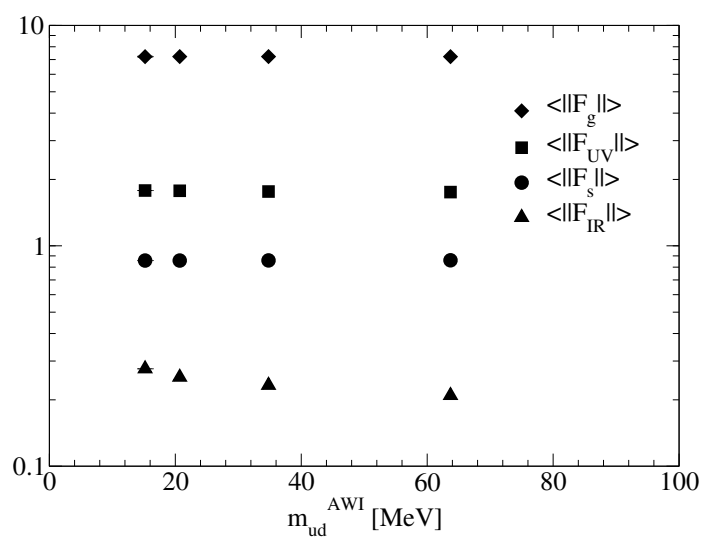

Figure 2: Magnitude of each force term as a function of the AWI quark mass $m_{\mathrm{ud}}^{\mathrm{AWI}}$. The errors are within symbols.

where the renormalization factors $Z_{A, P}$ and the improvement coefficients are determined perturbatively up to one-loop level. The results are given in Table 1 in physical units together with the corresponding pseudoscalar masses. It is encouraging that LDDHMC allows a simulation at $m_{\mathrm{ud}}^{\mathrm{AWI}}=15 \mathrm{MeV}$ which is roughly a quarter of the lightest quark mass employed in the previous CP-PACS/JLQCD project. In Table 1 we also give the results for $m_{\mathrm{PS}}^{2} / m_{\mathrm{ud}}^{\mathrm{AWI}}$, whose small quark mass dependence indicates that the finite volume effects are not sizable.

\subsection{Spectral gap distribution}

Recently Del Debbio et al. have discussed the relevance of the spectral gap distribution of the Wilson-Dirac operator for the stability of two-flavor lattice QCD simulations[9]. This study is applied to our three flavor case. We are interested in the effects of the strange quark contributions.

Following Ref.[9] we use the hermitian operator $Q_{m}=\gamma_{5} D_{m}$ rather than the Wilson-Dirac operator $D_{m}=(1 / 2)\left\{\gamma_{\mu}\left(\nabla_{\mu}^{*}+\nabla_{\mu}\right)-a \nabla_{\mu}^{*} \nabla_{\mu}\right\}+m_{0}$. The spectral gap is defined as

$$
\mu=\min \left\{|\lambda| \mid \lambda \text { is an eigenvalue of } Q_{m}\right\} .
$$

In Fig. 3 we show histograms of the spectral gap $\mu$ for our four values of $\kappa_{\text {ud }}$, which are obtained by the implicitly restarted Lanczos algorithm[10]. We observe roughly symmetric distributions for all the quark masses. Their median and average given in Table 1 are consistent with each other within error bars. In Fig.4 we plot the quark mass dependencies for $\mu_{\text {median }}$ and $\mu_{\text {average. Although }}$ both quantities are roughly proportional to the AWI quark mass, we find a clear tendency that they deviate upwards from the linearity in terms of $m_{\mathrm{ud}}^{\mathrm{AWI}}$ toward the chiral limit.

For the width of the distribution $\sigma$ we employ the same definition as in Ref.[9]: $\sigma$ is defined as $(v-u) / 2$, where $[u, v]$ is the smallest range of $\mu$ which contains more than $68.3 \%$ of the data. This is to avoid potentially large statistical uncertainties which might occur when data are not sufficiently sampled. The results of $\sigma$ are given in Table 1 . We find that the width of the distribution diminishes as the up and down quark mass decreases. An intriguing quantity is the combination $\sigma \sqrt{V} / a$ : In the two-flavor case Del Debbio et al. found that its value is roughly unity independent of the quark mass, volume, and lattice spacing[9]. In our three-flavor case, on the contrary, we find $\sigma \sqrt{V} / a<1$ and its value decreases as the up and down quark mass decreases. This fact may suggest that the 

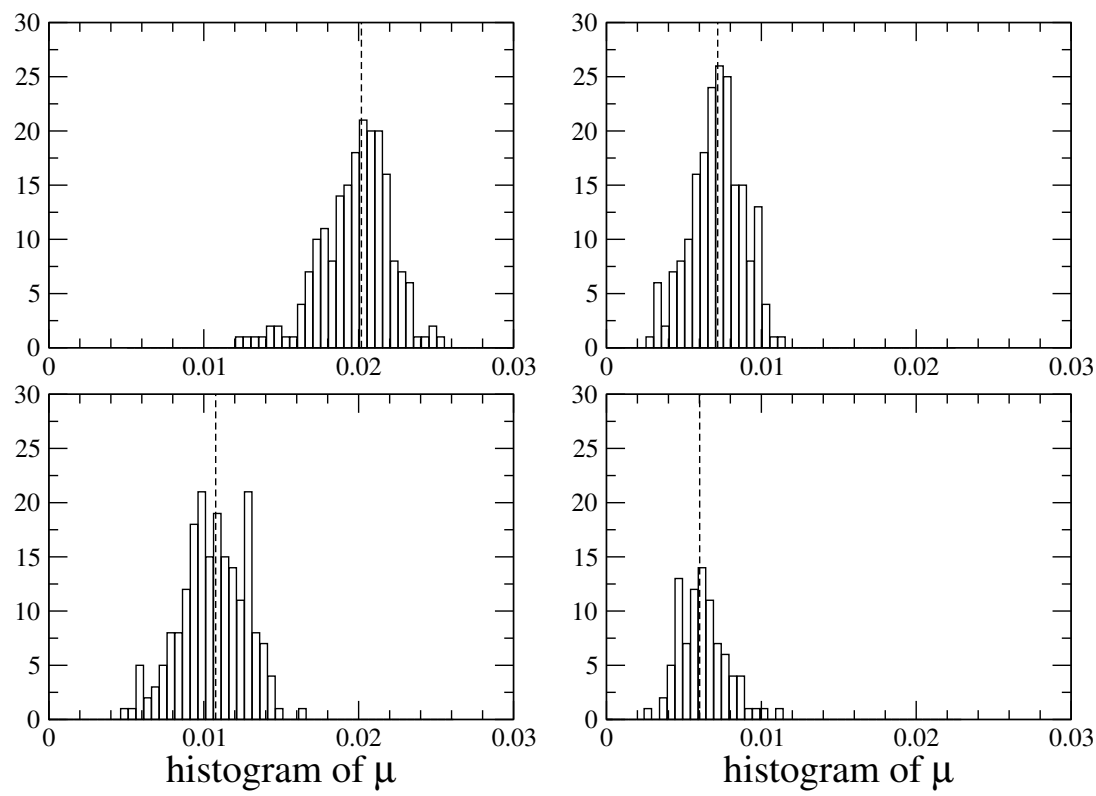

Figure 3: Histograms of $\mu$ for $\kappa_{\mathrm{ud}}=0.13700$ (left top), 0.13741 (left bottom), 0.13759 (right top) and 0.13770 (right bottom). Vertical dashed lines denote $\mu_{\text {median }}$.

contributions of the strange quark stabilize the simulation at the lighter quark masses by shrinking the gap distribution of the Wilson-Dirac operator.

\section{Plan for production run on PACS-CS}

We employ the LDDHMC algorithm with the replay trick for the up and down quarks. The Wilson-Dirac quark matrix inversion on the full lattice is accelerated by the SAP+GCR solver[11], which is three times faster than the BiCGStab algorithm for our parameter range[11]. For the strange quark we use the UV-filtered PHMC algorithm, which is two to three times more efficient than the PHMC algorithm[12].

We choose $\beta=1.83,1.90,2.05$ which are the same as in the previous CP-PACS/JLQCD project[1]. The physical lattice volume is fixed at $(3.0 \mathrm{fm})^{3}: 24^{3} \times 48$ for $\beta=1.83,32^{3} \times 64$ for $\beta=1.90$ and $40^{3} \times 80$ for $\beta=2.05$. Assuming that the relation $\sigma \propto a / \sqrt{V}$ found in Ref.[9] for the two-flavor simulation is applicable to the three-flavor case, we expect that a stable simulation at $m_{\mathrm{ud}}^{\mathrm{AWI}}=4 \mathrm{MeV}$ is possible at $\beta=1.90$ and even smaller up and down quark masses are accessible at $\beta=2.05$. Two strange quark masses are employed to interpolate the results on the physical point. We plan to accumulate 100 independent configurations with $10^{4}$ trajectories.

The first step of the project is a simulation with the up and down quark mass down to $m_{\mathrm{ud}}^{\mathrm{AWI}}=$ $7 \mathrm{MeV}$, which is about twice the physical mass and one order smaller than the lightest quark mass employed in the previous CP-PACS/JLQCD project. Once this is achieved, we can almost remove the systematic errors associated with the chiral extrapolation which are currently one of the most significant errors in lattice QCD simulations. The hadron spectrum together with the quark masses should be determined precisely. We are now preparing a nonperturbative renormalization factor for the quark masses employing the Schrödinger functional method. 


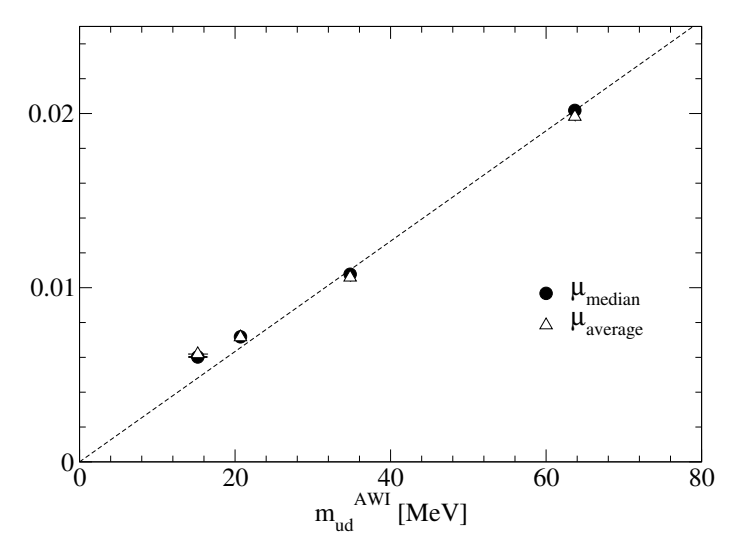

Figure 4: Median and average of the gap distribution as a function of AWI quark mass $m_{\mathrm{ud}}^{\mathrm{AWI}}$. The dashed line drawn from the origin to $\mu_{\text {median }}$ at the largest quark mass is to guide the eye.

There are two physical subjects which are especially interesting for the light quark masses. One is physics associated with topology. The CP-PACS/JLQCD Collaborations presented an encouraging result for the $\eta^{\prime}$ meson mass[13]. An intriguing point is that the signals become cleaner as the up and down quark mass decreases. The other is the $I=0 \pi-\pi$ scattering. In Ref.[14] we discussed that the light dynamical quarks are essential ingredients to study it.

This work is supported in part by Grants-in-Aid for Scientific Research from the Ministry of Education, Culture, Sports, Science and Technology (Nos. 13135204, 13135216, 15540251, 16540228, 16740147, 17340066, 17540259, 18104005, 18540250, 18740130).

\section{References}

[1] CP-PACS and JLQCD Collaborations, T. Ishikawa et al., these proceedings, PoS (LAT2006) 181.

[2] Y. Iwasaki, preprint, UTHEP-118 (Dec. 1983), unpublished.

[3] PACS-CS Collaboration, A. Ukawa et al., these proceedings, PoS (LAT2006) 039.

[4] M. Lüscher, JHEP 0305 (2003) 052; Comput. Phys. Commun. 165 (2005) 199.

[5] L. Giusti, these proceedings.

[6] JLQCD Collaboration, S. Aoki et al., Phys. Rev. D65 (2002) 094507.

[7] JLQCD Collaboration, S. Aoki et al., Phys. Rev. D73 (2006) 034501.

[8] A. Kennedy, Nucl. Phys. B (Proc. Suppl.) 140 (2005) 190.

[9] L. Del Debbio et al., JHEP 0602 (2006) 011.

[10] D. C. Sorensen, SIAM J. Matrix Anal. Appl. 13 (1992) 357.

[11] M. Lüscher, Comput. Phys. Commun. 156 (2004) 209.

[12] PACS-CS Collaboration, K.-I. Ishikawa et al., these proceedings, PoS (LAT2006) 027.

[13] CP-PACS and JLQCD Collaborations, T. Yoshié et al., these proceedings, PoS (LAT2006) 204.

[14] M. Fukugita et al., Phys. Rev. D52 (1995) 3003. 